\title{
Invariance for Impulsive Control Systems ${ }^{1}$
}

\author{
F. Lobo Pereira, ${ }^{*}$ G. Nunes Silva, ${ }^{* *}$ and V. Oliveira*** \\ * University of Porto, Porto, Portugal \\ ** University of British Columbia, Vancouver, Canada, \\ *** University of Campinas, Campinas, Brasil \\ Received January 16, 2007
}

\begin{abstract}
The conventional concepts of invariance are extended in this article to include impulsive control systems represented by measure driven differential inclusions. Invariance conditions and some of their main features are derived. The solution concept plays a critical role in the extension of the conditions for conventional problems to the impulsive control context.
\end{abstract}

PACS number: 02.30.Yy

DOI: $10.1134 / \mathrm{S} 0005117908050068$

\section{INTRODUCTION}

This article concerns the characterization of weak and strong invariance for impulsive control systems cast in the differential inclusion paradigm. In other words, given an impulsive dynamic system $(D)$ and a set $S$ containing its initial state value, we derive conditions ensuring that either at least one trajectory or all the trajectories remain in the set. The dynamic system $(D)$ is specified as follows:

$$
\text { (D) }\left\{\begin{array}{l}
d x(t) \in F(t, x(t)) d t+\mathbf{G}(t, x(t)) \mu(d t), \forall t \in[0, \infty) \\
x(0) \in C_{0}
\end{array}\right.
$$

where $F:[0, \infty) \times \mathbb{R}^{n} \hookrightarrow \mathcal{P}\left(\mathbb{R}^{n}\right), \mathbf{G}:[0, \infty) \times \mathbb{R}^{n} \hookrightarrow \mathcal{P}\left(\mathbb{R}^{n \times q}\right)$ are given set-valued functions and the control measure $\mu \in C^{*}([0, \infty) ; K)$, the set of measures (dual of the space of continuous functions) supported on the subsets of $[0, \infty)$ and with range in $K \subset \mathbb{R}^{q}, \mathcal{P}\left(\mathbb{R}^{n}\right)$ denotes the collection of subsets of $\mathbb{R}^{n}$, the set $K$ is a pointed cone, and $C_{0} \subset \mathbb{R}^{n}$ is a closed set.

Remark that the paradigm considered here is substantially more general than the usual measure driven differential equation studied in the literature,

$$
d x=f(t, x, u) d t+g(t, x) d \mu, \quad u(t) \in \Omega,
$$

since now, (1.1) may encompass some problems for which the singular dynamics may also depend on a certain control variable.

The main idea underlying our approach consists in defining a conventional control system equivalent to (1.1) by considering a natural time reparametrization, and, then, expressing the invariance conditions in terms of the data of the original problem.

A key role is played by the adopted solution concept (see, for example, [1,2], for finite time problems) which implies that any jump of a well defined solution to the measure-driven differential inclusion has to be such that there exists a path joining its endpoints that satisfies the singular dynamics. In this article, we consider an infinite time horizon version of this solution concept.

Impulsive systems has been regarded as a natural control paradigm in modeling, analysis and synthesis for a wide range of application areas, such as finance, management of renewable resources,

\footnotetext{
1 This work has been presented on the Symposium "Generalized Solution in Control Problems."
} 
and aerospace navigation, (consider [3-7], to name just a few references). This explains why a considerable body of theory for this class of systems (see, for example, [1,2,8-19], and references therein) and supporting control strategies computation schemes, [20-23] have been developed so far.

The research reported in this paper is part of a greater effort to understanding the features of impulsive dynamical systems governed by measure differential inclusions. In this vein, a previous work, [24], presented an extension of the Lyapunov stability theory to these systems. The basic condition was that the Lyapunov function composed with any trajectory of system had to be strictly decreasing to zero as time evolved to infinity. This Lyapunov condition was relaxed to alow for Lyapunov functions that were decreasing in "average" [25]. Roughly, this means that the value of the Lyapunov function along the state trajectory might increase in a jump, but this increase has to be compensated by the absolutely continuous evolution as time goes to infinity. In other words, there is no need for strict monotony.

Another general motivation for the development of impulsive control theory is due to the perceived relevance of the impulsive control paradigm for hybrid systems, [26,27]. The current dramatic technological developments in computation and communications have been fueling the engineering design of advanced controlled systems - say, for example, involving multiple autonomous devices and vehicles which are able to coordinate their activities to achieve the system's goals while satisfying given performance requirements - whose state evolution is dictated by the interaction of conventional time-driven dynamics and event-driven dynamics, and, thus, are naturally modeled in a hybrid systems context. By considering $x=\operatorname{col}(y, z)$, a certain index set $A$, and $\mathcal{Z}=\left\{z_{\alpha}: \alpha \in A\right\}$, we can see that the impulsive system

$$
\left\{\begin{array}{l}
\dot{y}=f(y, z, u), \quad u \in \Omega \\
d z=g(y, z) d \mu
\end{array}\right.
$$

models an hybrid system defined by the collection of conventional systems $\left\{\dot{y}=f_{\alpha}(y, u), u \in \Omega\right.$ : $\alpha \in A\}$, with $f_{\alpha}(y, u):=f\left(y, z_{\alpha}, u\right)$, whose discrete dynamics are specified by the singular component of the impulsive control system.

This article is organized as follows: in the next section we introduce the solution concept for infinite time horizon impulsive dynamic control systems and some associated pertinent results. Then, together with the presentation of relevant preliminary conventional definitions, we present, in Section 4, both weak and strong invariance conditions as well as the proofs of the main results. In section four, we illustrate the usage of the proposed invariance conditions with two simple examples, before concluding the article with some observations and remarks.

\section{SOLUTION CONCEPT AND BASIC RESULTS}

We adopt the concept of solution introduced in $[2,24]$ modified in order to accommodate the unbounded time interval $[0, \infty)$. Robustness is one of the key features of this concept. One advantage of this property concerns the fact that it enables the construction of a sequence of conventional control processes approximating, in a certain sense, the given reference impulsive process, and, therefore, it is a powerful instrument to extend results from control theory for conventional systems.

In order to present the solution concept, we need to introduce a change of time variable technique. Moreover, this reparametrization procedure is also of independent interest as it plays a critical role in our methods of proof. In order to define it, let, for $i=1, \ldots, q, M_{i} \in B V^{+}([0, \infty) ; \mathbb{R})$, be given 
by $M_{i}(t)=\int_{[0, t]} \mu_{i}(d s)$, for $t>0$ with $M_{i}(0)=0$, and consider:

$$
\begin{gathered}
\eta(t):=t+\sum_{i=1}^{m} M_{i}(t), \\
\bar{\eta}(t):=\left\{\begin{array}{lll}
\{\eta(t)\} & \text { if } \bar{\mu}(\{t\})=0 \\
{\left[\eta\left(t^{-}\right), \eta(t)\right]} & \text { if } \bar{\mu}(\{t\})>0 .
\end{array}\right.
\end{gathered}
$$

Here and in what follows, the space of absolutely continuous functions and the space of functions of bounded variation from $[0, \infty)$ to $\mathbb{R}^{n}$ are, respectively, denoted by $A C\left([0, \infty) ; \mathbb{R}^{n}\right)$ and $B V^{+}\left([0, \infty) ; \mathbb{R}^{n}\right), \bar{\mu}$ denotes the total variation of the measure $\mu$, i.e., $\bar{\mu}(d t):=\sum_{i=1}^{q} \mu_{i}(d t), \mathcal{L} \times \mathcal{B}$ is the product $\sigma$-field, where $\mathcal{L}$ denotes the Lebesgue subsets of $[0, \infty)$ and $\mathcal{B}$ denotes the Borel subsets of $\mathbb{R}^{q}, B$ is the open unit ball in the Euclidean space.

The above defined function $\eta$ is a reparametrization of the time variable $t$. Now, we introduce the notion of graph completion for the vector-valued measure $\mu$.

Definition 2.1. A family of graph completions associated to the vector-valued measure $\mu$ is the set of the pairs $\left(\theta, \gamma_{\mu}\right):[0, \infty) \rightarrow[0, \infty) \times K$, where $\theta:[0, \infty) \rightarrow[0, \infty)$ is the "inverse" of $\bar{\eta}:[0, \infty) \hookrightarrow$ $\mathcal{P}([0, \infty))$ in the sense that

$$
\theta(s)=t, \quad \forall s \in \bar{\eta}(t)
$$

and $\gamma_{\mu}:[0, \infty) \rightarrow \mathbb{R}^{q}$ is defined $\forall s \in \bar{\eta}(t), \forall t \in[0, \infty)$, by

$$
\gamma(s):= \begin{cases}M(\theta(s)) & \text { if } \bar{\mu}(\{t\})=0 \\ M\left(t^{-}\right)+\int_{\eta\left(t^{-}\right)}^{s} v(\sigma) d \sigma & \text { if } \bar{\mu}(\{t\})>0\end{cases}
$$

for some $v(\cdot) \in V^{t}$, where $t=\theta(s)$. Here, $M(\cdot):=\operatorname{col}\left(M_{1}(\cdot), \ldots, M_{q}(\cdot)\right)$ and

$$
V^{t}:=\left\{v: \bar{\eta}(t) \rightarrow K \mid \dot{\theta}(s)+\sum_{i=1}^{m} v_{i}(s)=1, \forall s \in \bar{\eta}(t), \int_{\bar{\eta}(t)} v(s) d s=\mu(\{t\})\right\} .
$$

Finally, we introduce the concept of robust solution.

Definition 2.2. The trajectory $x$, with $x(0)=x_{0}$, is admissible for $(1.1)$ if $x(t)=x_{a c}(t)+x_{s}(t)$ $\forall t \in[0, \infty)$, where

$$
\left\{\begin{array}{l}
\dot{x}_{a c}(t) \in F(t, x(t))+\mathbf{G}(t, x(t)) w_{a c}(t) \text { a.e. } \\
x_{s}(t)=\int_{[0, t]} g_{c}(\tau) w_{c}(\tau) d \bar{\mu}_{s c}(\tau)+\int_{[0, t]} g_{a}(\tau) d \bar{\mu}_{s a}(\tau) .
\end{array}\right.
$$

Here, $\bar{\mu}$ is the total variation measure associated with $\mu, \mu_{s c}, \mu_{s a}$ and $\mu_{a c}$ are, respectively, the singular continuous, the singular atomic, and the absolutely continuous components of $\mu, w_{a c}$ is the time derivative of $\mu_{a c}, w_{s c}$ is the Radon-Nicodym derivative of $\mu_{s c}$ with respect to its total variation, $g_{c}(\cdot)$ is a $\bar{\mu}_{s c}$ measurable selection of $\mathbf{G}(\cdot, x(\cdot))$ and $g_{a}(\cdot)$ is a $\bar{\mu}_{s a}$ measurable selection of the multifunction

$$
\tilde{G}\left(t, x\left(t^{-}\right) ; \mu(\{t\})\right):[0, \infty) \times \mathbb{R}^{n} \times K \hookrightarrow \mathcal{P}\left(\mathbb{R}^{n}\right) .
$$

The value of this multifunction is the set of all

$$
\zeta=\frac{\xi(\eta(t))-x\left(t^{-}\right)}{\bar{\mu}_{s a}(\{t\})}
$$


for which the process $\left(\xi(\cdot), \gamma_{\mu}(\cdot), v(\cdot)\right)$ satisfies:

$$
\begin{aligned}
& \left\{\begin{array}{l}
\dot{\xi}(s)=G(t, \xi(s)) v(s) \quad v(s) \in V^{t}, \quad \text { a.a. } s \in \bar{\eta}(t), \\
\dot{\gamma}_{\mu}(s)=v(s),
\end{array}\right. \\
& \left\{\begin{array}{l}
\xi\left(\eta\left(t^{-}\right)\right)=x\left(t^{-}\right) \\
\gamma_{\mu}\left(\eta\left(t^{-}\right)\right)=\mu([0, t))
\end{array}\right.
\end{aligned}
$$

for some measurable selection $G \in \mathbf{G}$ continuous in $t$ and Lipschitz in $x$. Here, $\left(\xi, \gamma_{\mu}\right) \in A C([0, \infty)$; $\left.\mathbb{R}^{n} \times \mathbb{R}^{q}\right)$, and the pair $\left(\theta, \gamma_{\mu}\right)$ is a graph completion of $\mu$.

Notice that we treat the trajectories of (1.1) as path-valued functions. This means that their images are curves in $\mathbb{R}^{n}$, for each time $t$. We denote such a trajectory by $x_{t}(\cdot)$. When $t$ is a continuity point of the control measure $\mu, x_{t}(\cdot)$ is a singleton $\left(x\left(t^{-}\right)=x\left(t^{+}\right)=x(t)\right)$, while if $t$ is in $M_{a}$, the support of the atomic component of the control measure, $\mu$ (i.e., $\mu(\{t\}) \neq 0 \forall t \in M_{a}$ and $\left.\mu(\{t\})=0, \forall t \in[0, \infty) \backslash M_{a}\right), x_{t}(\cdot)$ is regarded as a set of curves:

$$
x_{t}(\cdot):=\left\{\xi: \bar{\eta}(t) \rightarrow \mathbb{R}^{n} \mid \xi \text { satisfies }(2.1),(2.2)\right\} .
$$

In the light of the adopted solution concept, we will need the following definition.

Definition 2.3. Given a control process $(x, \mu)$, we say that $x$, a robust solution to (1.1) (in the sense of Definition 2.2), takes all its values in set $S$ if and only if:

- $x(t) \in S$ for all $t \in[0, \infty)$, and

- for each $t \in M_{a}$, we have that $\xi_{t}(s) \subset S$, for all $s \in \bar{\eta}(t)$.

For a given control measure $\mu$ and selections $f$ and $G$ of, respectively, $F$ and $\mathbf{G}$, the nonuniqueness of the trajectory as specified by Definition 2.2 results from the nonuniqueness of the graph completions of the vector-valued control measure $\mu$ defined above and from the fact that the vector fields associated with the singular dynamics (given by the columns of the selections of $\mathbf{G}$ ) are not assumed to be commutative (see [9]).

By using the time reparametrization technique, we can define conventional differential inclusions associated with the given impulsive differential inclusion. In a first instance, Theorem 2.1 below, the control measure is fixed and a family of particular conventional differential inclusion, indexed by the graph completions of the measure, is defined. A more elaborated result that allows for the measure being a choice variable (a desired feature) is stated afterwards in Theorem 2.2.

Theorem 2.1. Suppose that the multi-functions $F$ and $\mathbf{G}$ satisfy:

- $F$ takes closed sets as values and is $\mathcal{L} \times \mathcal{B}$-measurable,

- $\mathbf{G}$ takes closed sets as values and is Borel-measurable.

Fix a measure $\mu \in C^{*}([0, \infty) ; K)$ and a initial value $x_{0}$. Let $\left(\theta, \gamma_{\mu}\right)$ be the graph completion of $\mu$ and $\eta$ the reparameterization function.

(i) Suppose that $x(\cdot) \in B V^{+}\left([0, \infty) ; \mathbb{R}^{n}\right)$ is a robust solution to (1.1) (with respect to $\mu$ and $x_{0}$ ). Then, there is a solution, $y(\cdot) \in A C\left([0, \infty) ; \mathbb{R}^{n}\right)$, to

$$
\left\{\begin{array}{l}
\dot{y}(s) \in F(\theta(s), y(s)) \dot{\theta}(s)+\mathbf{G}(\theta(s), y(s)) \dot{\gamma}_{\mu}(s) \\
y(0)=x_{0}
\end{array}\right.
$$

for which

$$
x(t)=y(\eta(t)) \quad \text { for all } t \in(0, \infty) .
$$

Conversely, 
(ii) Suppose that $y(\cdot) \in A C\left([0, \infty) ; \mathbb{R}^{n}\right)$ is a solution to $(2.3)$. Then, there exists a solution $x(\cdot) \in B V^{+}\left([0, \infty) ; \mathbb{R}^{n}\right)$ to $(1.1)$ for which $(2.4)$ is satisfied.

(iii) Take a solution $x$ to (1.1). Let $y$ be a solution to (2.3) such that (2.4) is satisfied. Then,

$$
\|x\|_{B V} \leq\|y\|_{B V} .
$$

Proof. The proof is similar to that of a similar result (see Theorem 4.1 of [1]) in which the control measure is scalar-valued and, therefore, we omit it.

In the sequel, we will denote functions and variables of the extended reparametrized system by , i.e., we have $\dot{\tilde{x}} \in \tilde{F}(\tilde{x})$ where $\tilde{x}=\operatorname{col}\left(x^{0}, y\right)$ and

$$
\tilde{F}:=\left\{\operatorname{col}\left(v_{0}, F v_{0}+\mathbf{G} v\right): \operatorname{col}\left(v_{0}, v\right) \in \bar{V}\right\},
$$

where $\bar{V}=\left\{w \in \mathbb{R}^{q+1}: \sum_{k=1}^{q+1} w_{k}=1\right\}$. In this context $\tilde{S}=[0, \infty) \times S$.

Theorem 2.2. Assume that $F$ and $\mathbf{G}$ are Borel measurable.

If $(x, \mu)$ is a feasible control process for (1.1), then there exists a trajectory $\tilde{x}$ to

$$
\left\{\begin{array}{l}
\dot{\tilde{x}}(s) \in \tilde{F}(\tilde{x}(s)) \\
x^{0}(0)=0, \quad \text { with } \quad x^{0}(s) \rightarrow \infty \quad \text { as } \quad s \rightarrow \infty \\
y(0)=x_{0} .
\end{array}\right.
$$

Conversely, for each trajectory $\tilde{x}$ of (2.5), there exists an admissible control process $(x, \mu)$ to $(1.1)$ for which $x(t)=y(\eta(t))$.

Proof. The proof of this result is found in [25].

\section{INVARIANCE RESULTS}

In this section, we state the invariance results for the class of impulsive control systems considered in the previous sections.

A setup usually considered for invariance results in the context of conventional dynamic control systems, involves a closed set $S \subset \mathbb{R}^{n}$ and a set-valued map $F$ on the $(t, x)$ space specifying the dynamics in a differential inclusion form. The system $(F, S)$ is said invariant when at least one trajectory (weak) or all trajectories (strong) of $F$ initiated in $S$ will remain in this set for all future times.

By using Definition 2.3, the extension of the concepts of invariance to impulsive control systems is straightforward and are presented next. In these, we will denote by $((F, \mathbf{G}), S)$, the system composed by a set $S \subset \mathbb{R}^{n}$ and the measure driven differential inclusion specified by the set valued maps $F$ and $\mathbf{G}$.

Definition 3.1. We say that the system $((F, \mathbf{G}), S)$ is weakly invariant if, $\forall x_{0} \in S$, there exists a feasible control process $(x(\cdot), \mu(\cdot))$ to $(1.1)$, with $x(0)=x_{0}$, such that $x(\cdot)$ takes all its values in the set $S$.

Definition 3.2. The system $((F, \mathbf{G}), S)$ is strongly invariant if, $\forall x_{0} \in S$, all the feasible control process $(x(\cdot), \mu(\cdot))$ to $(1.1)$ with $x(0)=x_{0}$ are such that $x(\cdot)$ takes all its values in the set $S$.

Definition 3.3. The attainable set $\mathcal{A}\left(x_{0} ; T\right)$ at the time $T$ starting from $x_{0}$ at time 0 is given by

$$
\mathcal{A}\left(x_{0} ; T\right):=\left\{x(T):(x, \mu) \text { is a feasible process of }(1.1) \text { with } x(0)=x_{0}\right\} .
$$


In order to simplify the presentation, we will consider that set-valued maps $F$ and $\mathbf{G}$ depend only on the state variable. The results presented in this section require a set of hypotheses on these maps, designated by Standing Hypotheses, which we will assume to hold hereafter. In this context, they are stated as follows:

(H1) For every $x \in \mathbb{R}^{n}, F(x)$ and $\mathbf{G}(x)$ are convex, compact and non-empty sets.

(H2) The set-valued maps $F$ and $\mathbf{G}$ are upper semicontinuous.

(H3) There are constants $a$ and $b$ such that, for every $x \in \mathbb{R}^{n}$,

if $v \in F(x)$ then $\|v\| \leq a\|x\|+b$, and

if $V \in \mathbf{G}(x)$, then $\|V\| \leq a\|x\|+b$.

The hypothesis (H3) is known as linear growth condition.

We recall that $F$ is upper semicontinuous at $x$ if, given any $\varepsilon>0$, there exists $\delta>0$ such that

$$
\|y-x\|<\delta \Longrightarrow F(y) \subset F(x)+\varepsilon B .
$$

Before stating some equivalent forms to the weak invariance of the system $((F, \mathbf{G}), S)$, a result that generalizes the one for conventional control problems to the impulsive context, we will introduce the following technical assumption on the first component of the extended trajectory $\tilde{x}$ of the extended system,

$$
x^{0}(0)=0 \quad \text { and } \quad \lim _{s \rightarrow \infty} x^{0}(s)=\infty .
$$

Notice that this condition just means that the total variation of the control measure does not become unbounded in any time subinterval. Remark also that this condition is naturally satisfied if the following property of total variation measure $\bar{\mu}$ of the control measure $\mu$ holds:

$$
\forall T>0, \forall t \geq 0, \bar{\mu}([t, t+T])<\infty \quad \text { and } \quad \lim _{t \rightarrow \infty} \bar{\mu}([t, t+T])=0 .
$$

Proposition 3.1. The system $(\tilde{F}, \tilde{S})$ is weakly invariant if and only if the system $((F, \mathbf{G}), S)$ is also weakly invariant.

Proof. $[\Rightarrow]$ Suppose that the system $(\tilde{F}, \tilde{S})$ is weakly invariant. Let $x_{0} \in S$. Then, there exists a trajectory $\tilde{x}=\operatorname{col}\left(x^{0}, y\right)$ of $\tilde{F}$ such that $x^{0}(0)=0, y(0)=x_{0} \in S$ and $\tilde{x}(s) \in \tilde{S}, \forall s \geq 0$. By Theorem 2.2, there exists a process $(x, \mu)$ of $(F, \mathbf{G})$ such that $x(t)=y(\eta(t)), \forall t \geq 0$. That implies that $x(t) \in S, \forall t \geq 0$. Let $\left\{t_{i}\right\}$ be a sequence of the times supporting the atoms of $\mu$. By construction of $x$ (see [25]), we have that $y(s) \in x_{t_{i}}(s)$, for all $s \in \eta\left(t_{i}\right)$. Thus, we may conclude that $((F, \mathbf{G}), S)$ is weakly invariant.

$[\Leftarrow]$ Let $x_{0} \in S$. If $((F, \mathbf{G}), S)$ is weakly invariant, then there exists a feasible process $(x, \mu)$ of $(1.1)$ with $x(0)=x_{0}, x(t) \in S, \forall t \geq 0$, and, by denoting by $M_{a}$ the support of the atomic component of the control measure $\mu, \forall t \in M_{a}, \exists \xi_{t}(\cdot) \in x_{t}(\cdot)$ such that $\xi_{t}(s) \in S$, for all $s \in \bar{\eta}(t)$. By Theorem 2.1, there exists $\operatorname{col}\left(x^{0}, y\right)$, a solution of $(2.5)$, satisfying

$$
y(s)= \begin{cases}x(\theta(s)), & \text { if } s \in[0, \infty) \backslash \bigcup_{t \in M_{a}} \bar{\eta}(t) \\ \xi_{t}(s), & \text { if } s \in \bigcup_{t \in M_{a}} \bar{\eta}(t) .\end{cases}
$$

Hence, we have $\tilde{x}(s) \in \tilde{S}, \forall s \geq 0$ and, therefore, $(\tilde{F}, \tilde{S})$ is weakly invariant.

In order to present the next result, we need the definition of proximal normal cone (see [28] for more details and properties). Let $S \subset \mathbb{R}^{n}$ be a closed set and denote by $d_{S}(y)$ the distance function from $y$ to $S$ defined by $d_{S}(y):=\inf _{s \in S}\{\|y-s\|\}$. 
Definition 3.4. Let $x_{0} \in S$. $\zeta \in \mathbb{R}^{n}$ is a proximal normal vector to $S$ at $x_{0}$ if and only if $\exists \alpha>0$ such that

$$
d_{S}\left(x_{0}+\alpha \zeta\right)=\alpha\|\zeta\|
$$

The proximal normal cone to $S$ at $x_{0}, N_{S}^{p}\left(x_{0}\right)$, is the set of all proximal normals of $S$ at $x_{0}$.

Theorem 3.1. Suppose that the condition (3.1) holds. Then,

$$
\left\{\begin{array}{r}
\forall \tilde{x} \in \tilde{S}, \exists\left(v_{0}, v\right) \in \bar{V}, \exists(f, G) \in(F(x), \mathbf{G}(x)) \text { such that } \\
\left\langle\left(v_{0}, v_{0} f+\sum_{i=1}^{q} v_{i} g_{i}\right), \tilde{\zeta}\right\rangle \leq 0, \forall \tilde{\zeta} \in N_{\tilde{S}}^{p}(\tilde{x}) \\
\quad \text { if and only if } \\
\text { the system }((F, \mathbf{G}), S) \text { is weakly invariant. }
\end{array}\right.
$$

Proof. The proof of this result follows the structure of the corresponding result for conventional systems in [28].

From this reference, it can be shown that the following sufficient condition for (3.2) in terms of the Bouligand tangent cone $T_{\tilde{S}}^{B}(\tilde{x})^{2}$ holds

$$
\tilde{F}(x) \cap \operatorname{co} T_{\tilde{S}}^{B}(\tilde{x}) \neq \emptyset, \quad \forall \tilde{x} \in \tilde{S}
$$

which, in turn, is implied by

$$
\tilde{F}(x) \cap T_{\tilde{S}}^{B}(\tilde{x}) \neq \emptyset, \quad \forall \tilde{x} \in \tilde{S} .
$$

Observe that, since $T_{\tilde{S}}^{B}(\tilde{x})=\mathbb{R} \times T_{S}^{B}(x),(3.5)$ is equivalent to

$$
\bar{F}(x) \cap T_{S}^{B}(x) \neq \emptyset, \forall x \in S,
$$

where

$$
\bar{F}:=\left\{F v_{0}+G v: \operatorname{col}\left(v_{0}, v\right) \in \bar{V}\right\} .
$$

It can also be easy concluded that $N_{\tilde{S}}^{p}(\tilde{x})=\{0\} \times N_{S}^{p}(x)$ and, therefore, (3.2) is equivalent to

$$
\begin{aligned}
& \exists\left(v_{0}, v\right) \in \bar{V}, \exists(f(x), G(x)) \in(F(x), \mathbf{G}(x)) \quad \text { such that } \\
&\left\langle v_{0} f+\sum_{i=1}^{q} v_{i} g_{i}, \zeta\right\rangle \leq 0, \quad \forall \zeta \in N_{S}^{p}(x), \quad \forall x \in S .
\end{aligned}
$$

Clearly, from the definition of weak invariance, it can be easily concluded that (3.3) is equivalent to

$$
\forall x_{0} \in S, \forall \varepsilon>0, \exists \delta \in(0, \varepsilon) \text { such that } \mathcal{A}\left(x_{0} ; \delta\right) \cap S \neq \emptyset .
$$

Therefore, in order to complete the proof, we only need to show that (3.2) implies (3.3) and that (3.9) implies (3.5).

Now let us start with the first implication. Consider the system $(\bar{F}(x), S)$, and let $x_{0} \in S$. From (3.2), and, obviously (3.8), it follows that

$$
h(x, \zeta) \leq 0, \quad \forall \zeta \in N_{S}^{p}(x),
$$

\footnotetext{
${ }^{2}$ The Bouligand tangent cone to $S$ at $x$ defined as$$
T_{S}^{B}(x):=\left\{\lim _{i \rightarrow \infty} \frac{x_{i}-x}{\lambda_{i}}: x_{i} \stackrel{S}{\rightarrow} x, \lambda_{i} \downarrow 0\right\},
$$

where $x_{i} \stackrel{S}{\rightarrow} x$ means $x_{i} \in S$ and $x_{i} \rightarrow x$. 
where $h(x, \zeta):=\min \{\langle w, \zeta\rangle: w \in \bar{F}\}$. This allows us to use Theorem 4.2.4 of [28] to find a trajectory $y(\cdot)$ of $\bar{F}$ in $[0, \infty)$ such that $y(0)=x_{0}$ and $y(s) \in S$ for all $s \geq 0$. Now, we only have to construct a process $(x, \mu)$ of $(1.1)$ with $x(t)=y(\eta(t))$ for a suitable function $\eta$ such that $x(0)=x_{0}$, $x(t) \in S, \forall t \geq 0$, and $\xi_{t}(s) \in S, \forall s \in \bar{\eta}(t)$, for any $t \in M_{a}$, the support of the atomic component of $\mu$, and for some function $\xi_{t}(\cdot) \in x_{t}(\cdot)$. This can be done as in Proposition 3.1 and, therefore the system $((F, \mathbf{G}), S)$ is weakly invariant.

Next, we show that (3.5) follows from (3.9) or, after the remark earlier in this proof, its lower dimensional equivalent (3.6). First, notice that (3.3) is equivalent to (3.9). So, by Proposition 3.1, we can conclude that the claim (3.9) holds also to the attainable set for the reparameterized system

$$
\dot{y}(s) \in F(y(s)) \dot{\theta}(s)+\mathbf{G}(y(s)) \dot{\gamma}_{\mu}(s) .
$$

This allows us to adapt the proof of the similar result for the conventional control problem in [28]. Suppose that (3.9) holds for the reparameterized system and denote its attainable set by $\mathcal{A}^{y}\left(x_{0}, t\right)$. Then, $\forall n \in \mathbb{N}, \exists \delta_{n} \in(0,1 / n)$ with $\mathcal{A}^{y}\left(x_{0}, \delta_{n}\right) \cap S \neq \emptyset$. Therefore, for every $n$,

$$
\dot{y}_{n}(s) \in F\left(y_{n}(s)\right) \dot{\theta}_{n}(s)+\mathbf{G}\left(y_{n}(s)\right) \dot{\gamma}_{n}(s) \subset\left\{F\left(y_{n}(s)\right) v_{0}+\mathbf{G}\left(y_{n}(s)\right) v:\left(v_{0}, v\right) \in \bar{V}\right\}=\bar{F}\left(y_{n}(s)\right) .
$$

Here, $\left(\theta_{n}, \gamma_{n}\right)$ is a graph completion of the measure $\mu_{n} \in C^{*}([0, \infty) ; K)$.

The functions $y_{n}, n=1, \ldots$, have the same Lipschitz constant $K$, so that

$$
\frac{\left\|y_{n}\left(\delta_{n}\right)-x_{0}\right\|}{\delta_{n}} \leq K, \quad \forall n
$$

Thus, subsequence (no relabeling) can be chosen to assert the existence of $v \in \mathbb{R}^{n}$ such that $v:=\lim _{n \rightarrow \infty} \frac{y_{n}\left(\delta_{n}\right)-x_{0}}{\delta_{n}}$. That is, $v \in T_{S}^{B}\left(x_{0}\right)$. Then, we need only to show that $v \in \bar{F}\left(x_{0}\right)$ in order to deduce (3.5). We can write

$$
y_{n}\left(\delta_{n}\right)-x_{0}=\int_{0}^{\delta_{n}} \dot{y}_{n}(s) d s .
$$

We have that $\bar{F}$ is upper semi-continuous. Let $\varepsilon \geq 0$. Then, for $n$ sufficiently large, we have that

$$
y_{n}\left(\delta_{n}\right)-x_{0} \in \int_{0}^{\delta_{n}}\left\{\widetilde{F}\left(x_{0}\right)+\varepsilon B\right\} d s .
$$

By dividing by $\delta_{n}$ and passing to the limit when $n \rightarrow \infty$ we obtain

$$
v \in \bar{F}\left(x_{0}\right)+\varepsilon B \text {. }
$$

The result follows from the arbitrariness of $\varepsilon$.

Proposition 3.2. The system $(\tilde{F}, \tilde{S})$ is strongly invariant if and only if the system $((F, \mathbf{G}), S)$ is also strongly invariant.

Proof. $[\Rightarrow]$ Let $(x, \mu)$ a feasible process for $(1.1)$ such that $x(0) \in S$. Then, by Theorem 2.1, there exists a trajectory $y$ for $F(y) \dot{\theta}(s)+\mathbf{G}(y) \dot{\gamma}(s) \subset \bar{F}(y)$ (the multifunction defined in (3.7)) such that $y(0)=x(0) \in S, y(s)=x(\theta(s))$ for all $s \in[0, \infty) \backslash \bigcup_{t \in M_{a}} \bar{\eta}(t)$, and $y(s) \in x_{t}(s)$ for all $t \in M_{a}$, being $M_{a}$ the support of the atomic component of $\mu$.

Let $x^{0}(s)=\theta(s)$ and notice that $\tilde{x}=\operatorname{col}\left(x^{0}, y\right)$ is a trajectory for $\tilde{F}$ satisfying $\tilde{y}(0) \in \tilde{S}$. Since $(\tilde{F}, \tilde{S})$ is strongly invariant, we have that $y(s) \in S$ for all $s \geq 0$. Since, for any $t \in M_{a}, y(s)$ can, at the interval $\bar{\eta}(t)$, be taken to be any curve in $x_{t}$ that satisfies $(2.1),(2.2)$, we reach the conclusion that $x_{t}(\cdot) \subset S$ for all $t \in M_{a}$. Therefore, it follows from $x(t)=y(\eta(t))$ that $x_{t}(\cdot) \subset S$ for all $t \geq 0$, i.e., $((F, \mathbf{G}), S)$ is strongly invariant. 
$[\Leftarrow]$ Let $\tilde{x}$ be an arbitrary trajectory of $\tilde{F}$ with $\tilde{x}(0) \in \tilde{S}$. We will show that, for any arbitrary $T>0, \tilde{x}(T) \in \tilde{S}$.

By Theorem 2.2, an admissible process $(x, \mu)$ of $(F, \mathbf{G})$ on $[0, T]$, with $x(0)=y(0) \in S$ can be constructed.

Let $T^{*}:=\eta(T)$. By assumption, $((F, \mathbf{G}), S)$ is strongly invariant, and, therefore, $x_{t}(\cdot) \subset S$ for all $t \in[0, T]$. By construction, we have that $y(s)=x(\theta(s))$ for all $s \in\left[0, T^{*}\right] \backslash \bigcup_{t \in M_{a}} \bar{\eta}(t)$ and $y(s) \in x_{t}(s)$ for all $s \in \bar{\eta}(t)$. Then, $y(s) \in S$ for all $s \in\left[0, T^{*}\right]$. But $T^{*} \geq T$ and this implies that $y(T) \in S$. Thus $(\tilde{F}, \tilde{S})$ is strongly invariant, since $\tilde{x}$ is an arbitrary solution of $(2.5)$.

In the next result, that is a generalization of the similar result for conventional dynamic control systems (see, e.g., [28]), we need the Lipschitz condition for set-valued functions. We say that a set-valued function $\Gamma: \mathbb{R}^{n} \hookrightarrow \mathbb{R}^{m}$ is locally Lipschitz if for each $x_{0} \in \mathbb{R}^{n}$, there exist $\delta, K>0$ such that

$$
\Gamma(x) \subset \Gamma(y)+K\|x-y\| B \quad \forall x, y \in x_{0}+\delta B .
$$

Theorem 3.2. Suppose that $F$ and $\mathbf{G}$ are locally Lipschitz. Then,

$$
\begin{gathered}
\left\{\begin{array}{c}
\forall \tilde{x} \in \tilde{S}, \forall\left(v_{0}, v\right) \in \bar{V}, \text { we have, } \forall \tilde{\zeta} \in N_{\tilde{S}}^{P}(\tilde{x}) \\
\max _{(f, G) \in(F(x), \mathbf{G}(x))}\left\langle\tilde{\zeta}, v_{0} f+\sum_{i=1}^{q} v_{i} g_{i}\right\rangle \leq 0
\end{array}\right. \\
\text { if and only if } \\
\text { the system }((F, \mathbf{G}), S) \text { is strongly invariant. }
\end{gathered}
$$

Remark. Following the arguments in [28] it is straightforward to show that alternative equivalent characterizations of strong invariance are:

(a) $\tilde{F}(x) \subset T_{\tilde{S}}^{C}(\tilde{x}) \forall \tilde{x} \in \tilde{S} ;^{3}$

(b) $\tilde{F}(\tilde{x}) \subset T_{\tilde{S}}^{B}(\tilde{x}) \forall \tilde{x} \in \tilde{S}$;

(c) $\tilde{F}(\tilde{x}) \subset \operatorname{coT}_{\tilde{S}}^{B}(\tilde{x}) \forall \tilde{x} \in \tilde{S}$;

(d) $\forall x_{0} \in S, \exists \varepsilon>0$, such that $\mathcal{A}\left(x_{0} ; t\right) \subset S \forall t \in[0, \varepsilon]$.

Proof. It follows from Theorem 4.3.8 of [28] that (3.10) is a necessary and sufficient condition for the strong invariance of the system $(\tilde{F}, \tilde{S})$ (as well as conditions (a)-(d) in the above remark). Then, the conclusion follows immediately from Proposition 3.2.

\section{EXAMPLES}

In this section we present two examples which illustrate the main features of our invariance conditions for impulsive control systems.

$\overline{{ }^{3} T_{S}^{C}(x), \text { denotes the }}$ Clarke tangent cone to $S$ at $x$, which is given by:

$$
T_{S}^{C}(x):=\left\{v \in \mathbb{R}^{n}: d_{S}^{\circ}(x ; v) \leq 0\right\}
$$

where $d_{S}^{\circ}(x ; v)$ is the generalized directional derivative of $d_{S}(\cdot)$ at $x$, in the direction $v$. This is defined by

$$
d^{\circ}(x ; v):=\limsup _{y \rightarrow x ; t \downarrow 0} \frac{d_{S}(y+t v)-d_{S}(y)}{t} .
$$




\subsection{Example 1}

Let $x=\operatorname{col}\left(x_{1}, x_{2}\right) \in \mathbb{R}^{2}$ and consider the system $((F, \mathbf{G}), S)$ defined by

- $F(x)=\left\{\left(\left|x_{2}\right|-u-\frac{1}{2}, u\right):-1 \leq u \leq 1\right\}$;

- $\mathbf{G}(x)=\left\{\left(-\frac{x_{1}}{\left|x_{1}\right|}, k\right): k \geq 0\right\} ;$ and

- $S=\left\{\left(x_{1}, x_{2}\right):-2 \leq x_{1} \leq 2\right\}$.

In order to write down the invariance conditions for this system, we start by computing the proximal normal cone of $S$.

$$
N_{S}^{p}\left(x_{1}, x_{2}\right)= \begin{cases}\left\{\left(\lambda \frac{x_{1}}{\left|x_{1}\right|}, 0\right): \lambda \geq 0\right\}, & \text { if } x_{1}= \pm 2 \\ \{(0,0)\}, & \text { otherwise. }\end{cases}
$$

In order to conclude that inequality (3.8) holds, and, thus, by Theorem 3.1, the weak invariance of the system, take $\left(v_{0}, v_{1}\right)=(0,1) \in \bar{V}$. It follows that, for all values of $u$,

$$
\left\langle v_{0}\left(\left|x_{1}\right|-u-\frac{1}{2}, u\right)+v_{1}\left(-\frac{x_{1}}{\left|x_{1}\right|}, k\right),\left(\lambda \frac{x_{1}}{\left|x_{1}\right|}, 0\right)\right\rangle=\left\langle\left(-\frac{x_{1}}{\left|x_{1}\right|}, k\right),\left(\lambda \frac{x_{1}}{\left|x_{1}\right|}, 0\right)\right\rangle=-\lambda .
$$

Now, let us examine in detail the case with $u=k=1$. In this case, the trajectories are given by

$$
\left\{\begin{array}{l}
x_{1}(t)=\int\left|x_{2}(t)\right| \mathrm{d} t-\frac{3}{2} t+A \\
x_{2}(t)=t+B
\end{array}\right.
$$

where $A, B$ are arbitrary constants that can be found by initial conditions. Take $\mu(\mathrm{d} t):=\delta_{t_{i}}(t) \mathrm{d} t$ as the control measure, where $\delta_{t_{i}}$ is the Dirac impulse at $t=t_{i}$, being $\left\{t_{i}: i \in \mathbb{N}\right\}$, the sequence of times in $M_{a}$, the support of the atomic component of the control measure such that either (i) $x_{1}\left(t_{i}\right)=-2$ and $-\frac{3}{2} \leq x_{2}\left(t_{i}\right) \leq \frac{3}{2}$, or (ii) $x_{1}\left(t_{i}\right)=2$.

Conditions (i) and (ii) above are such that the state trajectory hits the boundary of $S$ in a region where the non-singular field "points outwards" the set $S$. At these times, the singular field forces the trajectory to jump into the interior of $S$. The paths specifying the jumps at $t_{i}$ are given by

$$
\left(\xi_{1}(s), \xi_{2}(s)\right)= \begin{cases}\left(s-2-\eta\left(t_{i}^{-}\right), s+x_{2}\left(t_{i}^{-}\right)-\eta\left(t_{i}^{-}\right)\right) & \text {if } x_{1}\left(t_{i}^{-}\right)=-2 \\ \left(-s+2+\eta\left(t_{i}^{-}\right), s+x_{2}\left(t_{i}^{-}\right)-\eta\left(t_{i}^{-}\right)\right) & \text {if } x\left(t_{i}^{-}\right)=2\end{cases}
$$

where $\eta$ is the time reparametrization function defined in Section 2. Since $\eta\left(t_{i}\right)-\eta\left(t_{i}^{-}\right)=1$, we have that $\xi_{1}\left(\eta\left(t_{i}\right)\right)=\operatorname{sgn}\left(x_{1}\left(t_{i}^{-}\right)\right)$, for $\left.x_{1}\left(t_{i}^{-}\right)\right)= \pm 2$. Figure 1 below shows the non-singular field in $S$ and a typical trajectory starting at $\operatorname{col}(-2,-3)$.

(a)

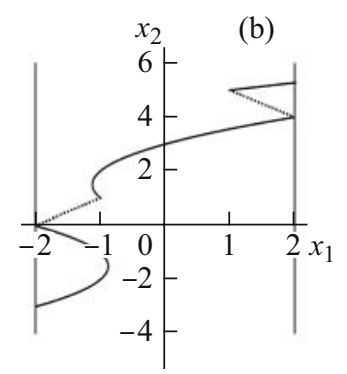

Fig. 1. The non-singular field in (a) $S$ and (b) a trajectory: the dotted segments represent the "jump paths." 


\subsection{Example 2}

Now we consider the invariance characterization of the system $((F, \mathbf{G}), S)$ defined by

- $x=\operatorname{col}\left(x_{1}, x_{2}\right)$ and $S=\left\{x: x_{1}^{2}+x_{2}^{2} \leq 9\right.$ and $\left.x_{2} \leq 2\right\}$,

- $F(x)=\{A x\}, \mathbf{G}(x)=\{B x\}$, with $A=\left[\begin{array}{rr}\alpha & -2 \\ 1 & 0\end{array}\right]$ and $B=\left[\begin{array}{rr}-\beta & 0 \\ 0 & -\beta\end{array}\right]$, where $\alpha, \beta$ are in $\mathbb{R}$, with $\beta \neq 0$.

We will show that (3.8) is satisfied for all $x \in S$ and, thus, by Theorem 3.1, the given system is weakly invariant.

Let us choose $\alpha=2$ and $\beta=1$. The computation of the proximal normal cone is straightforward and yields

$$
N_{S}^{p}(x)= \begin{cases}\{(0,0)\}, & \text { if } x \in \operatorname{int} S \\ \left\{\lambda \zeta: \zeta_{1} \leq \frac{-\sqrt{5}}{2} \zeta_{2}, \zeta_{2}, \lambda \geq 0\right\}, & \text { if } x=(-\sqrt{5}, 2) \\ \left\{\lambda \zeta: \zeta_{1} \leq \frac{\sqrt{5}}{2} \zeta_{2}, \zeta_{1}, \lambda \geq 0\right\}, & \text { if } x=(\sqrt{5}, 2) \\ \{(0, \lambda): \lambda \geq 0\}, & \text { if } x \in\left\{\left(x_{1}, 2\right): \sqrt{5}<x_{1}<\sqrt{5}\right\} \\ \{2 \lambda x: \lambda \geq 0\}, & \text { otherwise. }\end{cases}
$$

Now we just have to assert that, for each $x \in S$ and $\zeta \in N_{S}^{P}(x)$, the existence of a feasible pair $\left(v_{0}, v_{1}\right)$, for which $\bar{h}\left(v_{0}, v_{1} ; \zeta, x\right):=\left\langle v_{0} f(x)+v_{1} g(x), \zeta\right\rangle \leq 0$. Clearly

$$
\bar{h}\left(v_{0}, v_{1} ; \zeta, x\right)\left\{\begin{array}{lll}
=0, & \text { if } x \in \operatorname{int} S, & \forall\left(v_{0}, v_{1}\right) \\
\leq-\frac{9}{2} \lambda \zeta_{2}, & \text { if } x=(-\sqrt{5}, 2), & \left(v_{0}, v_{1}\right)=(0,1) \\
\leq \frac{1-2 \sqrt{5}}{4} \lambda \zeta_{2}, & \text { if } x=(\sqrt{5}, 2), & \left(v_{0}, v_{1}\right)=\frac{1}{2}(1,1) \\
=-2 \lambda, & \text { if } x \in\left\{\left(x_{1}, 2\right): \sqrt{5}<x_{1}<\sqrt{5}\right\}, & \left(v_{0}, v_{1}\right)=(0,1) \\
=-2 \lambda\|x\|_{2}^{2}, & \text { if } x \in \partial S \cap\left\{\left(x_{1}, x_{2}\right): x_{2}<2\right\}, & \left(v_{0}, v_{1}\right)=(0,1),
\end{array}\right.
$$

where $\partial S$ is the boundary of $S$. Thus we have that (3.8) is satisfied for all points in $S$. Therefore, by Theorem 3.1 the system $((F, \mathbf{G}), S)$ is weakly invariant so that, for all $x_{0}=\operatorname{col}\left(x_{01}, x_{02}\right) \in S$, we are able to construct a process $(x, \mu)$ such that $x(0)=x_{0}, x(t) \in S, \forall t \geq 0$ and $\exists \xi \in x_{\tau}$ with $\xi(s) \in S$ if $\mu(\{\tau\})>0$.

In this case, $\alpha=2$ and $\beta=1$, the matrix $A$ has complex eigenvalues with positive real part, and so we have an unstable equilibrium point. Hence the trajectories converge far away from the origin when $t \rightarrow \infty$. Therefore, we need to use the singular vector field to prevent the trajectories leaving $S$. This is achieved by taking $\mu(\mathrm{d} t):=\sum_{i \in \mathbb{N}} l_{i} \delta_{t-t_{i}}(t)$ as the control measure, where $\left\{t_{i}: i \in \mathbb{N}\right\}$ is a sequence of times supporting the atoms of $\mu$ and $l_{i}$ is the area of the impulse at time $t_{i}$. Each $t_{i}$ is such that $x\left(t_{i}^{-}\right) \in \partial S$. The size of the jump must be chosen adequately for each $t_{i}$. We will do this in a way that the trajectory will jump to points in the set $\left\{x: x_{1}^{2}+x_{2}^{2} \leq 1\right\} \subset S$. If $x\left(t_{i}^{-}\right)$is the initial state, then, for each $i$, the trajectories of the singular field are given by

$$
\left\{\begin{array}{l}
\xi_{1}(t)=x_{1}\left(t_{i}^{-}\right) \exp \left(\eta\left(t_{i}^{-}\right)-t\right) \\
\xi_{2}(t)=x_{2}\left(t_{i}^{-}\right) \exp \left(\eta\left(t_{i}^{-}\right)-t\right)
\end{array}\right.
$$

In order to obtain $\xi_{1}^{2}\left(\eta\left(t_{i}\right)\right)+\xi_{2}^{2}\left(\eta\left(t_{i}\right)\right)=1$, we need to choose (recall that $l_{i}=\eta\left(t_{i}\right)-\eta\left(t_{i}^{-}\right)$)

$$
l_{i}=\frac{\ln \left(x_{1}\left(t_{i}^{-}\right)^{2}+x_{2}\left(t_{i}^{-}\right)^{2}\right)}{2} .
$$

Figure 2 shows a trajectory starting in $x_{0}=\operatorname{col}(0,-2)$. 
(a)

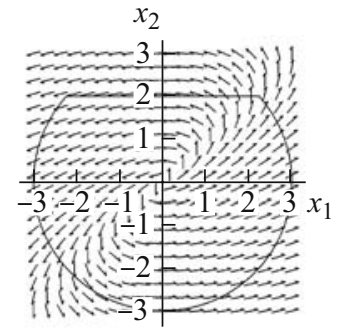

(b)

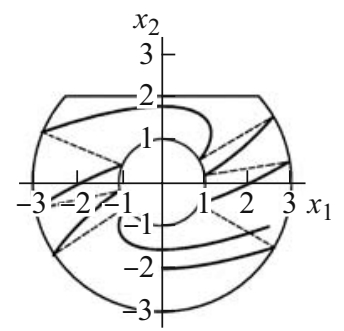

Fig. 2. The non-singular field in (a) $S$ and (b) a trajectory: the dashed parts represent the "jump paths."

We can also conclude that the system $((F, \mathbf{G}), S)$ is weakly invariant for other values of $\alpha$ and $\beta$. Actually, if $\beta>0$, we can, for any value of $\alpha$, construct, in a similar way, a process $(x, \mu)$ such that $((F, \mathbf{G}), S)$ is invariant. In particular, in some cases we do not need to activate the singular vector field. For example, if $\alpha=-\sqrt{8}$, the matrix $A$ has a double real negative eigenvalue and, therefore, the system is clearly invariant. But, if $\beta<0$, we cannot say the same for all $\alpha$ : if $\alpha=2$ both the conventional and the singular systems are unstable and we cannot construct a process $(x, \mu)$ such that the system $((F, \mathbf{G}), S)$ is invariant, for any selection $\left(v_{0}, v_{1}\right) \in \bar{V}$.

\section{CONCLUDING REMARKS}

In this work, we provided invariance conditions for impulsive control systems governed by measure driven differential inclusions. The invariance conditions presented here are a natural extension of ones for control systems with absolutely continuous dynamics which can be found, for example, in [28]. We also illustrated the application of these conditions with two examples revealing their essential features for impulsive control systems.

This is a first step in providing invariance for impulsive systems. A direct method that deals only with the impulsive system, without re-parametrization, is desired. This remains an open area of research.

\section{ACKNOWLEDGMENTS}

The authors are grateful for the support for the research project "Aplicações da Teoria do Controlo Impulsional e de Visão Computacional para Sistemas Robóticos Autonomos" funded under the GRICES-CAPES. The first author acknowledges the supported of FCT under the research project "Constrained Optimal Control and Applications," and the second author acknowledges the support of $\mathrm{CNPq}$ under the research project "Otimização Dinâmica e Análise de Sistema Impulsivos."

\section{REFERENCES}

1. Silva, G.N. and Vinter, R.B., Measure Differential Inclusions, J. Math. Anal. Appl., 1996, vol. 202, pp. $727-746$.

2. Pereira, F.L. and Silva, G.N., Necessary Conditions of Optimality for Vector-Valued Impulsive Control Problems, Syst. 85 Control Lett., 2000, vol. 40, pp. 205-215.

3. Clark, C., Clarke, F., and Munro, G., The Optimal Exploitation of Renewable Stocks, Econometrica, 1979, vol. 47, pp. 25-47.

4. Baumeister, J., On Optimal Control of a Fishery, in NOLCOS'01-5th IFAC Symposium on Nonlinear Control Systems, 2001. 
5. Gurman, V.I., Modeli upravleniya prirodnymi resursami (Models of Natural Resources Control), Moscow: Nauka, 1981.

6. Brogliato, B., Nonsmooth Impact Mechanics: Models, Dynamics and Control, Lect. Notes Control Inform. Sci., Berlin: Springer-Verlag, 1996, vol. 220.

7. Marec, J.P., Optimal Space Trajectories, New York: Elsevier, 1979.

8. Arutyunov, A., Jacimovic, V., and Lobo Pereira F., Second Order Necessary Conditions for Optimal Impulsive Control Problems, European Control Conference, 2001.

9. Bressan, A. and Rampazzo, F., Impulsive Control Systems with Commutative Vector Fields, J. Optim. Theory Appl., 1991, vol. 71, no. 1, pp. 67-83.

10. Bressan, A. and Rampazzo, F., Impulsive Control Systems without Commutativity Assumptions, J. Optim. Theory Appl., 1994, vol. 81, no. 3, pp. 435-457.

11. Dykhta, V., Impulse-trajectory Extension of Degenerate Optimal Control Problems, IMACS Ann. Comput. Appl. Math., 1990, vol. 8, pp. 103-109.

12. Dykhta, V.A. and Samsonyuk, O.N., Optimal Impulse Control with Applications, Moscow: Nauka, 2000.

13. Gurman, V.I., On Optimal Processes with Unbounded Derivatives, Autom. Remote Control, 1972, vol. 17 , pp. 14-21.

14. Kolokolnikova, G.A., Discontinuous Trajectories Optimality in the Nonlinear Optimal Control Problems, Proc. 13th World Congress of the IFAC, 1996, pp. 353-357.

15. Miller, B.M., The Generalized Solutions of Nonlinear Optimization Problems with Impulse Control, SIAM J. Control Optim., 1996, vol. 34.

16. Motta, M. and Rampazzo, F., Space-time Trajectories of Nonlinear Systems Driven by Ordinary and Impulse Controls, Diff. Integral Equat., 1995.

17. Rishel, R., An Extended Pontryagin Principle for Control Systems whose Control Laws Contain Measures, SIAM J. Control, 1965, vol. 3, pp. 191-205.

18. Vinter, R.B. and Pereira, F.M., A Maximum Principle for Optimal Processes with Discontinuous Trajectories, SIAM J. Control Optim., 1988, vol. 26, pp. 205-229.

19. Silva, G.N. and Vinter, R.B., Necessary Conditions for Optimal Impulsive Control Problems, SIAM J. Control Optim., 1997, vol. 35, pp, 1829-1846.

20. Dykhta, V. and Derenko, N., Numerical Methods based on Generalized Stationary Condition for Impulsive Control Problems, in Proc. Workshop Comput. Logics, Algebra Intelligence Control, Irkutsk, 1994, vol. 2, pp. 59-70.

21. Baturin, V. and Goncharova, E., Improvement Method based on Approximate Representation of Reachable Set, Autom. Remote Control, 1999, no. 11, pp. 19-29.

22. Baturin, V. and Ourbanovich, D., Approximate Optimal Control Methods based on Extension Principle, Novosibirsk: Nauka, 1997.

23. Gurman, V.I., Baturin, V.A., Moskalenko, A.I., et al., Methods of Improvement in Computational Experiments, Novosibirsk: Nauka, 1988.

24. Pereira, F.L. and Silva, G.N., Stability for Impulsive Control Systems, Dynam. Syst., 2002, vol. 17, no. 4, pp. 421-434.

25. Pereira, F.L. and Silva, G.N., Lyapunov Stability of Measure Driven Differential Inclusions, Diff. Uravn., 2004, vol. 40, no. 8, pp. 1059-1067.

26. Aubin, J.-P., Impulse Differential Equations and Hybrid Systems: A Viability Approach, Berkeley: Univ. of California, 2000.

27. Branicky, M., Borkar, V.S., and Mitter, S.K., A Unified Framework for Hybrid Control: Model and Optimal Control Theory, IEEE Trans. Automat. Control, 1998, vol. 43, no. 1, pp. 31-45.

28. Clarke, F.H., Ledyaev, Yu., Stern, R., and Wolenski, P., Nonsmooth Analysis and Control Theory, Graduate Texts in Mathematics, Springer-Verlag, 1998, vol. 178.

This paper was recommended for publication by V.N. Bukov, a member of the Editorial Board 